\title{
Editorische Vorbemerkung - Hinweise zur Benutzung
}

Die Wiedergabe von Handschrift im typographischen Satz ist auch bei einer noch so differenzierten Druckgestaltung nicht als Abbildung (,mimesis'), sondern eher als Resultat einer Übersetzung (,interpretatio') von einem polymorphen in ein stereotypes Schreibsystem zu verstehen. Das Schreiben und Lesen von Manuskripten toleriert Spielräume, die auch die Grenzen der genauesten Umschrift im Druck sprengen. Das betrifft sowohl ihre Gesamtkomposition als auch die Materialität schon jedes einzelnen Zeichens.

Jede Handschrift besitzt neben generellen auch situative, dem Schreibprozeß unterliegende Merkmale, die in der drucktechnischen Reproduktion fortfallen müssen. Was an einem Manuskript individuell ist, wird im Druck,typisiert'. Die Einmaligkeit des handschriftlichen Schreibflusses widersetzt sich jeder Manuskriptdokumentation, die auf normierte Druckbuchstaben angewiesen ist. Zu solchen nicht quantifizierbaren Phänomenen eines Manuskripts gehören, zum Beispiel, die Verteilung des Schriftquantums in der Zeile und auf der Seite, der mal penible, mal fahrige Schriftduktus, die variierende Größe der Buchstaben, die Abhängigkeit der Schriftfigur vom jeweiligen Schreibgerät, von der jeweiligen Aufschreibefläche, die schwankende Tendenz zu kalligraphischer Realisation oder privater Stenographie.

Bei den Nachlaßaufzeichnungen Nietzsches kommt hinzu, daß sie in ihrer überlieferten Gestalt keineswegs als Druckvorlage dienen sollten; ihr Schreiber konnte sich als ihr wahrscheinlich einziger Leser verstehen, das heißt er konnte private, für ihn selbstverständliche Weisen des Auf- und Nieder-, Ab-, Um-, Weiter- und Überschreibens realisieren.

So sind Zeichen für Flüchtigkeit oder Insistenz, Binnen- und Endverschleifungen, private Abkürzungen und Kürzel, Sonder- und Privatzeichen zwar in Nietzsches Manuskripten, nicht aber im Setzkasten für den Buchdruck vorhanden. Sie erschweren der Transkription, Befund, Deutung und Darstellung in Einklang zu bringen. Die Forderung nach der authentischen Umschrift klingt wie ein unerfüllbarer Imperativ, wenn, auch nach Jahren der Entzifferungspraxis, kein schlüssiges Kriterium dafür gefunden werden kann, ob ein graphematisch keinesfalls korrumpierter Schriftzug nun durch „unseren“, „unsern“ oder „unsren“ wiedergegeben werden soll. Es ließen sich gewichtigere Beispiele zuhauf nennen.

Nietzsches Handschrift der späten Jahre gilt als schwer lesbar; sie ist hochgradig individualisiert. Das Varianzspektrum einzelner Grapheme ist beträchtlich, ihre Differenzierbarkeit dagegen oft unzureichend. Polyvalente Einzelzeichen kommen ebenso vor wie nicht unterscheidbare Wortbilder mit offenkundig unterschiedlicher Bedeutung. Ein Wille zur Einheitlichkeit und Konformität ist kaum zu erkennen. Die nicht mundierten Aufzeichnungen sind zum Teil mehrfach, nicht selten unsystematisch und unvollständig überarbeitet. Die Niederschriften können als Material zur Relektüre für ihren Verfasser charakterisiert werden, der sein Schreiben offenbar als einen immer wieder neu nicht abschließbaren Prozeß empfand.

Die in der neunten Abteilung der Kritischen Gesamtausgabe transkribierten Manuskripte aus Nietzsches Nachlaß werden auf der jeweils mitgelieferten CD-ROM in digitalisierter Faksimilierung präsentiert. Dadurch ist der Vergleich der Transkription mit den handschriftlichen Aufzeichnungen gewährleistet. Angesichts dieser direkten Anschaulichkeit erübrigen sich weitgehend umfängliche Erklärungen genereller Transkriptions- und Darstellungskriterien.

Die Druckseite zeigt das farblich unterlegte Transkriptionsfeld (I) im jeweiligen Format des Manuskripts mit den von Nietzsches Hand stammenden Aufzeichnungen in differenzierter Umschrift. Die zum äußeren Seitenrand hin anschließende Spalte (II) bietet innenbündig Raum erstens für die Zeilenmarkierung, zweitens für die aus dem Transkriptionsfeld ausgegliederten, gleichfalls farblich unterlegten Manuskriptsegmente und drittens für Hinweise zu Anschlüssen bei nicht habituellem Schriftverlauf; außenbündig werden in dieser Spalte Notate und Markierungen fremder Hand verzeichnet. Am Fuß der Seite (III) werden die Druckorte aus KGW, KSA und KGB sowie kritische Anmerkungen zur Transkription lemmatisch mitgeteilt.

Diejenigen Seiten, die in den Bänden 12 und 13 nicht im Format der Manuskripte präsentiert werden (etwa Mp XVI, 33r), sind erkennbar durch ein über der rechten oberen Ecke des verkleinerten Transkriptionsfeldes platziertes Minus-Symbol. Das Originalformat der jeweiligen Seite wird im Nachbericht in der Beschreibung der Manuskripte mitgeteilt.

Manuskriptseiten, die nach Drehung des Heftes beschrieben sind, werden auch im Druck um $90^{\circ}, 180^{\circ}$ oder $270^{\circ}$ gewendet; das dreigegliederte Layout ist entsprechend angepaßt.

Die Seitenausrichtung der Manuskripte in den Bänden 12 und 13 beruht auf der Position der archivalisch vorgenommenen Foliierung. Wo in der editorischen Wiedergabe der Aufzeichnungen diese Ausrichtung im Uhrzeigersinn um $90^{\circ}, 180^{\circ}$ oder $270^{\circ}$ gedreht erscheint (etwa Mp XV, 75v), wird dies durch rechts über dem oberen Textfeldrand platzierte Dreh-Symbole angezeigt. 
Anders als bei Schriftzeichen läßt sich bei Seiten- und Passagenstreichungen, Randanstreichungen und Markierungen nicht immer entscheiden, ob diese von Nietzsche oder von späteren Bearbeitern herrühren. Für die Dokumentation solcher redaktioneller Spuren im Manuskript wurde folgende Differenzierung vorgenommen: stammen sie mit Sicherheit von Nietzsches Hand, werden sie im Transkriptionsfeld gezeigt; sind sie sicher oder mit großer Wahrscheinlichkeit einer fremden Hand zuzuordnen, werden sie in der Randspalte verzeichnet; bei unsicherer Herkunft werden sie zwar ins Transkriptionsfeld aufgenommen, ihre Zuschreibung in den Fußnoten jedoch in Frage gestellt.

Die Wiedergabe der Schriftverteilung auf den Manuskriptseiten (Ränder, Einzüge, Zeilenabstände etc.) ist nach Maßgaben des Drucks standardisiert; Aufzeichnungen mit Schriftzeilen, die im Transkriptionsfeld nicht Platz fänden, sind kondensiert, das heißt enger gesetzt.

Die Transkription unternimmt es nicht, die in den Aufzeichnungen wechselnde Deutlichkeit der Schrift abzubilden. Abkürzungen werden als solche wiedergegeben, die zahlreichen Ligaturen und Verschleifungen aber aufgelöst.

In deutscher Schreibschrift niedergeschriebene Passagen sind im Druck durch Antiquaschrift, in lateinischer Schreibschrift oder Druckschrift geschriebene Passagen sind in serifenloser Schrift gesetzt; wo diese Schriftarten in Nietzsches Handschrift in Abweichungen oder gemischt vorkommen, wird nur der jeweils vorherrschende Duktus berücksichtigt.

Die Verwendung verschiedener Schreibmittel wird im Druck durch unterschiedliche Farben dargestellt; es wird zwischen schwarzen, braunen und violetten Tinten sowie zwischen Blei-, Rot- und Blaustiften unterschieden. Bei der Vielzahl verwendeter Tinten und Stifte identifizieren die Druckfarben nicht ein einzelnes Schreibgerät oder -mittel, sondern zeigen deren jeweilige Unterscheidbarkeit an. Eine zusätzliche Druckfarbe (,grün') signalisiert einen differenzierbaren Korrekturvorgang mit einer bereits verwendeten Tintenfarbe; diese wird kurz als „Tinte der letzten Korrektur“ bezeichnet.

Um darüber hinaus einzelne Schreib- und Korrekturvorgänge zu unterscheiden, benutzt die Transkription verschiedene Schriftgrößen. Erste Niederschriften sind in normaler Größe wiedergegeben; als Einfügungen oder nicht selbständige Hinzufügungen gewertete Aufzeichnungen erscheinen in kleiner Schrift (petit); an diesen vorgenommene Änderungen oder Zusätze werden in einer noch kleineren Schrift gesetzt, falls dieser Vorgang nicht schon durch die Position der Aufzeichnung oder durch das differente Schreibmittel erkennbar ist.

Aufzeichnungen, die nicht entziffert werden konnten, werden durch eine Reihe von Kreuzen „xxxx““ dargestellt.

Graphische Elemente im Manuskript (Streichungen, Abtrennungslinien, Anschlußstriche, Einfügungs- und Fortsetzungsschlaufen, Zeichnungen etc.) sind stilisiert; insbesondere gibt die Transkription nur über die Häufigkeit, nicht aber über unterschiedliche Weisen der Tilgung einzelner Wörter oder Zeilen Auskunft; diagonale Passagen- und Seitenstreichungen werden als solche wiedergegeben, andere Formen (Parallel- oder Mehrfachstreichungen, Schraffur- oder Kreuzstreichungen etc.) werden als X-förmige Tilgung gezeigt.

Die Vielzahl der Manuskript-,Verschmutzungen' (Tintenflecke und -abdrücke, andere mit Sicherheit unabsichtliche, Verunreinigungen', auch von fremder Hand, etc.) läßt eine systematische Dokumentation nicht zu; wo Tintenabdrücke bereits zu Fehlentzifferungen geführt haben, wird dies unter den Berichtigungen im Nachbericht mitgeteilt.

II

Zeilenmarkierung: Für eine differenzierte Umschrift, die auch die typographischen Aspekte des Manuskripts berücksichtigt, ist eine regelrechte Zeilenzählung bezüglich vieler Manuskriptseiten oft nicht praktikabel, bisweilen unmöglich. Bei der hier vorgenommenen Zeilenmarkierung, die ausschließlich der Funktion einer bequemeren Referenz dient, sind die indizierten Zeilen mit geraden Zahlen bezeichnet; die dadurch eingeschlossenen Zwischenräume werden durch die entsprechenden, in der Zeilenzahlleiste nicht ausgedruckten ungeraden Zahlen repräsentiert.

Ausgegliederte Manuskriptsegmente: Die neben die Zeilenmarkierung plazierten, farblich unterlegten Segmente (etwa Mp XVI, 2r,12), die zum Transkriptionsfeld gehören, enthalten die im Manuskript durch Überschreibung getilgte Schrift (im Beispiel: „Die“). Sie korrespondieren mit den auf gleicher Zeilenhöhe im Transkriptionsfeld hervorgehobenen Segmenten, in denen die überschreibende Schrift (im Beispiel: „Zur“) wiedergegeben ist. Befinden sich mehrere dieser Segmente auf einer Höhe (etwa Mp XVI, 2r,6), so folgen sie einander entsprechend der Leserichtung.

Anschlußpfeile: Auf den unmittelbaren Fortgang des Schreibverlaufs, sofern dieser nicht offensichtlich ist, weisen als Lesehilfe Anschlußpfeile hin. Diese sind entweder optisch verfolgbar in den Seitenfalz gesetzt (etwa W II 10, 182,20 $\rightarrow$ 183,4 oder Z I 2, 12,46 $\rightarrow$ 13,30) oder außen an die Zeilenleiste mit entsprechender Zeilen- beziehungsweise Seiten- und Zeilenzahl (etwa Mp XIV, 418,3 $\rightarrow 418,1$ beziehungsweise Mp XIV, 417,46 $\rightarrow 418,2)$; so bedeutet zum Beispiel

$\rightarrow 1 \quad$ Fortsetzung auf Zeile 1 derselben Seite

$3 \rightarrow \quad$ Fortsetzung von Zeile 3 derselben Seite

$\rightarrow 418,2 \quad$ Fortsetzung auf Seite 418, Zeile 2

$417,46 \rightarrow \quad$ Fortsetzung von Seite 417, Zeile 46

Wenn der Fortgang der Aufzeichnung nicht vom unmittelbaren Zeilenende ausgeht oder nicht zum unmittelbaren Zeilenanfang führt, wird neben dem Lesepfeil der direkte Anschluß aus dem Transkriptionsfeld zitiert (etwa Mp XIV, 420,60 $\rightarrow$ 420,50).

Bearbeitungsspuren fremder Hände: Randanstreichungen (etwa Mp XVI, 89v,2) und (zumeist vertikal-zentrierte) Passagen- (etwa Mp XV, 78r,49-55) oder Seitenstreichungen (etwa Mp XIV, 416,2) werden unter Angabe der in der Transkription betroffenen Zeilen in der Randspalte außenbündig mitgeteilt. Markierungen wie „„“ (etwa Mp XV, 78r,2), „““ (etwa Mp XV, 78r, 12), „”“ (etwa W II 4, 116,9) 
oder „//“ (etwa W II 6, 138,3) und Kommentare oder andere Notizen (etwa Mp XIV, 417,1 oder Mp XV, 74v,3) sind transkribiert; den (ungefähren) Ort dieser Aufzeichnungen im Manuskript signalisiert ein ins Transkriptionsfeld gesetzter Platzhalter „,“. Nicht unter die verzeichneten Spuren fremder Hand werden die stereotypen Prozeduren von Paginierung und Foliierung aufgenommen; sie sind in der Beschreibung der Manuskripte dokumentiert.

III

In den Fußnoten werden die Druckorte für solche Aufzeichnungen angegeben, die als Vorlage für die „Nachgelassenen Fragmente“ in KGW VII 3 und KGW VIII 1-3, für die „Vorstufen“ und „Fassungen“ in KGW VII 4/2 und KSA 14 und für die in die Briefwechselausgabe übernommenen „Briefentwürfe“ in KGB interpretiert werden konnten. Wichtige frühere Druckorte der Aufzeichnungen sowie ihre Eingliederung in die Kompilation „Der Wille zur Macht“ werden in der „Konkordanz der Druckorte“ im Nachbericht mitgeteilt.

Ebenfalls in den Fußnoten werden kritische Anmerkungen zur Transkription mitgeteilt, deren Art und Anzahl sich auch subjektiver Entscheidung verdanken. Ist die vor dem Lemma stehende Zeilenzahl unterstrichen (etwa Mp XV, 81v, Anmerkung zu Zeile 63), so bezieht sich die Mitteilung auf die ausgegliederten Manuskriptsegmente der entsprechenden Zeile.

Mit „? wird auf unsichere Entzifferungen hingewiesen, mit „““ auf Verschreibungen und stark verschliffene oder „atypische“ Schreibweisen (durch Fettdruck hervorgehoben) und mit „Vk“ auf nachträgliche Verdeutlichungskorrekturen im Manuskript (ebenfalls durch Fettdruck hervorgehoben).

Schreibfehler werden nur dort nach „>“ von den Herausgebern korrigiert (beziehungsweise nach „>?“ bei einem aus mehreren möglichen Verbesserungen gewählten Vorschlag), wo sie als Lesehilfen nötig erscheinen; ebenso wird bei Auflösungen von Abkürzungen verfahren.

Insbesondere die bei Änderungen im Manuskript nicht systematisch durchgeführten grammatikalischen Anpassungen sind in diesen Anmerkungen nicht ergänzt; der Ausdruck „nach Korrektur des Kontextes > “ macht aber (etwa Mp XV, 81v, Anmerkung zu Zeile 56) auf den Umstand aufmerksam, daß ein im Manuskript nicht verbesserter Schriftzug dank seiner Gestalt schon als die von den Herausgebern mitgeteilte, dem korrigierten Kontext entsprechende Lesart interpretiert werden kann.

Um nicht die Kohärenz von Aufzeichnungen zu konstituieren, werden allenfalls thematisch naheliegende, von der Manuskriptgestalt her aber nicht gesicherte oder lediglich vermutete Fortsetzungen (etwa W II 7, 3, Anmerkung zu Zeile 36) nur in den Fußnoten mitgeteilt.

IV

Der auf der CD-ROM gegebene Nachbericht enthält die Beschreibung der Manuskripte, Querverweise zu den Abschreibprozessen, einen Stellenkommentar(inklusive eines Literaturverzeichnisses), Berichtigungen, eine Konkordanz der „Fragmente“ und „Briefentwürfe“, eine Konkordanz früherer Druckorte sowie einen Namenindex. Alle diese Mitteilungen entsprechen dem vorläufigen Erkenntnisstand und haben transitorische Gültigkeit. Sie sollen die Arbeit mit der Manuskriptdokumentation schon während des Zeitraums ihres Entstehens erleichtern. Der definitive Nachberichtband wird nach Abschluß der Manuskriptdokumentation publiziert.

$\mathrm{V}$

Ab den „Aufzeichnungen aus diversen Heften“ in KGW IX 11 enthalten einige dokumentierte Seiten Niederschriften (und Bearbeitungsspuren), die der Gesamtarchitektur von KGW zufolge nicht in die Abteilung IX gehören: Es handelt sich dabei um vor dem Frühjahr 1885 verfaßte Aufzeichnungen Nietzsches, um Passagen seiner Druckmanuskripte oder um an ihn gerichtete Briefe. Diese daher nicht transkribierten Aufzeichnungen werden unter Weglassung der farblichen Unterlegung an entsprechender Stelle im Textfeld als Mitteilungen der Herausgeber angezeigt (etwa N VI 8, 61).

VI

In der Transkription benutzte Schriften und Farben:

Schrift (Weidemann) deutsche Schreibschrift

Schrift (News Gothic) lateinische Schreibschrift oder Druckschrift

Schrift (Bodoni) deutsche Schreibschrift (Diktat Ns)

Schrift (Futura) lateinische Schreibschrift oder Druckschrift (Diktat Ns)

normal erste Niederschriften

petit $\quad$ Einfügungen und Zusätze

petit spätere Einfügungen und Zusätze

Schrift schwarze Tinte

Schrift Bleistift

Schrift braune Tinte

Schrift Rotstift

Schrift violette Tinte

Schrift Blaustift

Schrift "Tinte der letzten Korrektur“ 
In den Randspalten und Fußnoten benutzte Schriften:

Schrift (Frutiger Light) Zitate aus dem Transkriptionsfeld sowie fremde Hand Schrift (Frutiger Light Italic) Mitteilungen der Herausgeber

Zeichen und Abkürzungen:

$\begin{array}{ll}\mathrm{xxxxx} & \text { nicht entziffert } \\ ? & \text { unsichere Entzifferung } \\ \dot{c} & \text { „atypische“ Schreibweise } \\ > & \text { Korrektur } \\ >? & \text { Korrekturvorschlag } \\ V k & \text { Verdeutlichungskorrektur im Manuskript } \\ A n m . & \text { Anmerkung } \\ B e & \text { Briefentwurf } \\ K G W & \text { Kritische Gesamtausgabe, Werke } \\ K S A & \text { Kritische Studienausgabe, Werke } \\ K G B & \text { Kritische Gesamtausgabe, Briefe } \\ K S B & \text { Kritische Studienausgabe, Briefe } \\ M s & \text { Manuskript } \\ N & \text { Nietzsche }\end{array}$

In KGW gebräuchliche Siglen für Nietzsches Werke und Schriften:

GT Die Geburt der Tragödie

$U B \quad$ Unzeitgemässe Betrachtungen

DS David Strauss der Bekenner und der Schriftsteller

$H L \quad$ Vom Nutzen und Nachtheil der Historie für das Leben

$S E \quad$ Schopenhauer als Erzieher

WB Richard Wagner in Bayreuth

GMD Das griechische Musikdrama

ST Socrates und die Tragoedie

DW Die dionysische Weltanschauung

GG Die Geburt des tragischen Gedankens

$U Z \quad$ Ursprung und Ziel der Tragoedie

SGT Sokrates und die griechische Tragoedie

$B A \quad$ Ueber die Zukunft unserer Bildungsanstalten

$C V \quad$ Fünf Vorreden zu fünf ungeschriebenen Büchern

NJ Ein Neujahrswort an den Herausgeber der Wochenschrift „Im neuen Reich“

PHG Die Philosophie im tragischen Zeitalter der Griechen

WL Ueber Wahrheit und Lüge im aussermoralischen Sinne

$M D \quad$ Mahnruf an die Deutschen

MA Menschliches, Allzumenschliches

$V M \quad$ Vermischte Meinungen und Sprüche

WS Der Wanderer und sein Schatten

$M$

Morgenröthe

Idyllen aus Messina

Die fröhliche Wissenschaft

FWS „Scherz, List und Rache“

FWP Lieder des Prinzen Vogelfrei

$\mathrm{Za}$

Lieder des Prinzen Vogelf
Also sprach Zarathustra

Jenseits von Gut und Böse

Zur Genealogie der Moral

Der Fall Wagner

Götzen-Dämmerung

Nietzsche contra Wagner

Ecce homo

Der Antichrist

Dionysos-Dithyramben 